\title{
Cutaneous manifestations of Nilotinib
}

\author{
Deena Mudawi ${ }^{1}$, Nancy Kassem ${ }^{2}$, Abdulqadir Nashwan ${ }^{1}$ and Mohamed A Yassin ${ }^{1 *}$ \\ ${ }^{1}$ Hematology section, Medical Oncology, HMC \\ ${ }^{2}$ Department of pharmacy, NCCCR, HMC
}

Received: June 20, 2018; Accepted: August 1, 2018; Published: August 2, 2018

*Corresponding author: Mohamed A Yassin, Consultant Hematologist, NCCCR-HMC, E-mail: yassinmoha@gmail.com

\begin{abstract}
Nilotinib is a highly selective second-generation Bcr-Abl tyrosine kinaseInhibitor (TKIs), which is effective in patients with Philadelphia chromosome-positive chronic myeloid leukemia (CML). Tyrosine kinase inhibitors have broadly similar types of non-hematological adverse side effects (AES), but some of the AES are specific to one drug compared to the others. In general, the TKIs are well tolerated with mostly (grade1-2) toxicities, and relatively limited grade (34) toxicities. For best management of CML patients receiving TKI therapy, early recognition and knowledge of potential toxicities are required, allowing for optimal management of the condition without interruption of the drug.
\end{abstract}

Key words: Nilotinib; Skin rash; chronic myeloid leukemia

\section{Introduction}

Chronic myeloid leukemia is a clonal myeloproliferative disorder characterized by the expansion of hematopoietic cells carrying the Philadelphia chromosome $(\mathrm{Ph})$, resulting from a reciprocal translocation of the long arms of chromosomes 9 and 22.

A novel fusion gene is formed, $B C R-A B L$, which encodes a constitutively active protein tyrosine kinase. Nilotinib is a second-generation_Bcr-Abl, tyrosine kinase inhibitor introduced in 2007 for the treatment of chronic myeloid leukemia (CML) as front line or Imitanib resistance or intolerant (CML), and other malignancies. Nilotinib has a favorable safety profile, however is associated with several off-target side effects, including skin rashes, the incidence of which has been reported in clinical trials and ranges from $20 \%$ to $60 \%$,[13], but no specific type of rashes are mentioned.

Here we present two patients with different types of skin rashes, which developed two weeks after starting Niltiniob and disappeared with conservative treatment without stopping or modifying the dose of Niltoinib

\section{Case reports}

\section{Case 1}

31 -year-old Filipino male with no chronic illness, discovered incidentally to have leukocytosis and review of peripheral smear was suggestive of chronic myeloid leukemia. Clinical examination revealed splenomegaly $4 \mathrm{~cm}$ below costal margin and bone marrow biopsy with aspiration and conventional cytogenetics confirmed the diagnosis of chronic myeloid leukemia in chronic phase. The patient was started on Nilotinib (300 mg twice a day) as upfront therapy and two weeks after initiation of Nilitonib the patient developed extensive skin rash.

On examination, the patient had diffuse follicular papules over the trunk and upper limbs extending to the dorsum of both hands. Although the rash was diffuse, it was more covering the left side of his back and few p eruptions on the extensor portions of the upper limbs and the dorsum of both hands as well (Fig. 1, Fig. 2). The patient declined a skin biopsy. Nilotinib was continued without dose adjustment, emollients and low dose topical corticosteroid (1\%) hydrocortisones were prescribed, and seven days later the rash disappeared.

\section{Case 2}

26 -year-Chinese female with no chronic illness, presented with increase abdominal girth and was found to have leukocytosis with a peripheral smear suggestive of chronic myeloid leukemia. Clinical examination revealed significant splenomegaly $10 \mathrm{~cm}$ below costal margin, and bone marrow biopsy with aspiration and conventional cytogenetics confirmed the diagnosis of

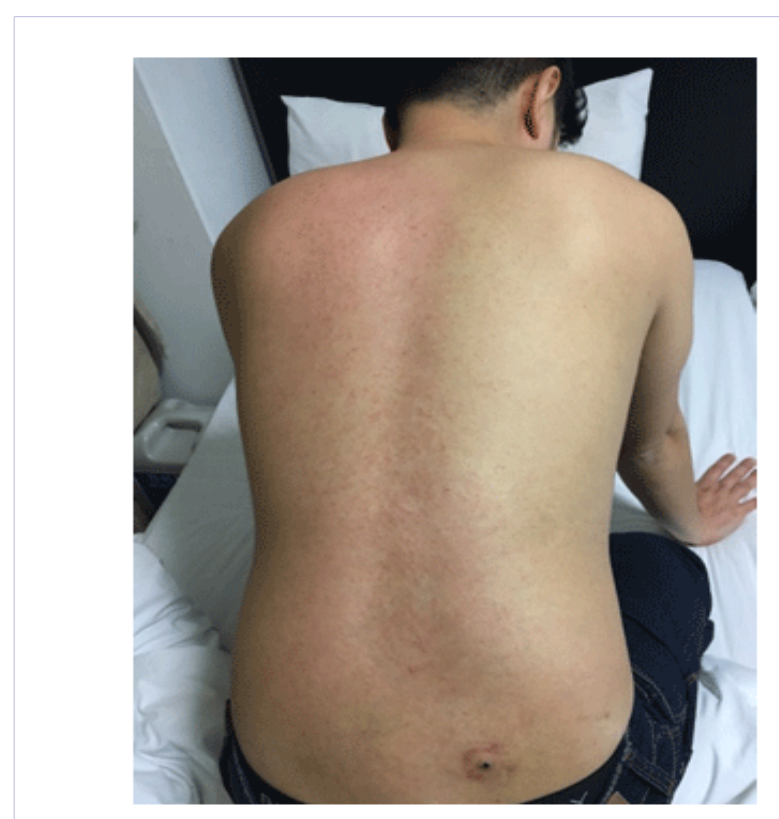

Figure 1 


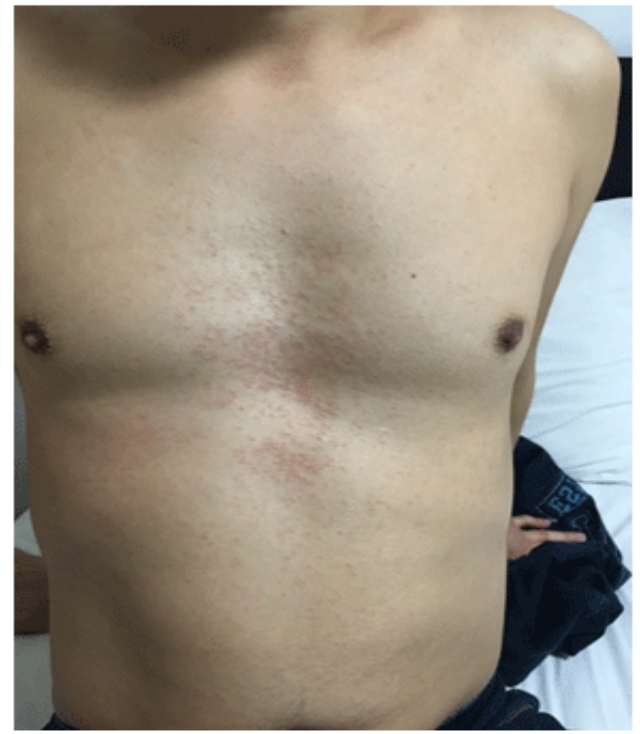

Figure 2

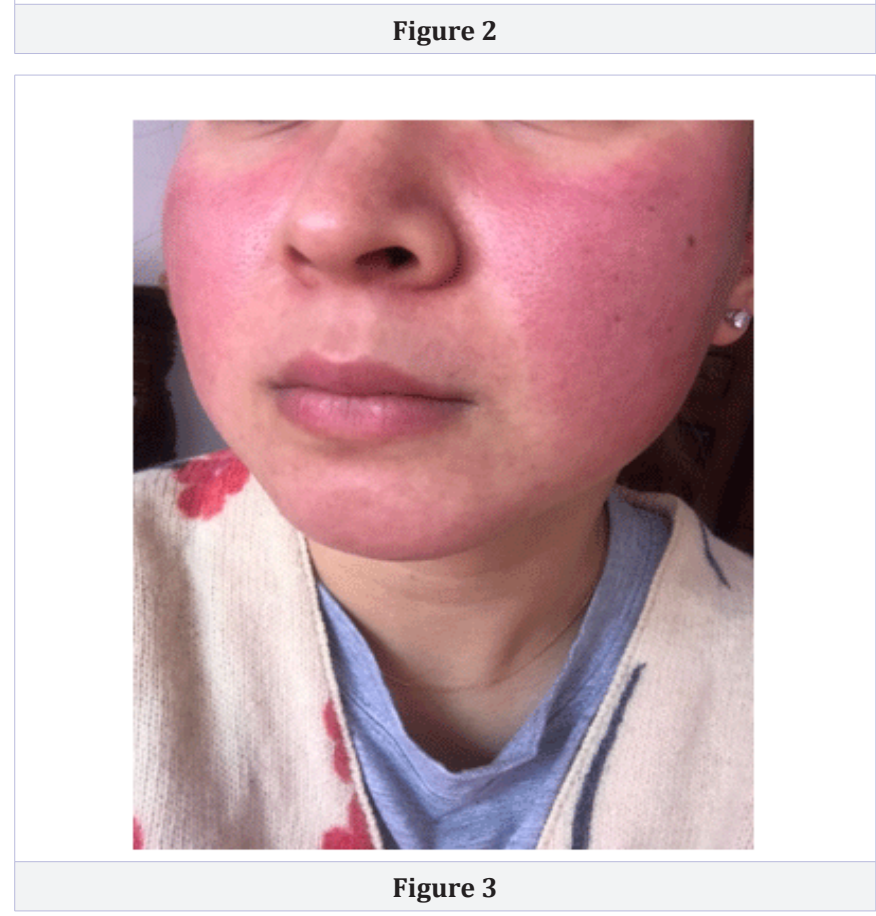

chronic myeloid leukemia in chronic phase. She was started on Nilotinib (300 mg twice a day) and two weeks after initiation of Nilotinib; the patient developed an extensive rash on her face that mimicked the classical butterfly skin rash of systemic lupus erythematosus. The differential diagnosis included auto-immune disease I.e.: systemic lupus erythematosus (SLE), however her ANA was negative and there was no clinical, hematological, or biochemical manifestations of autoimmune disease. Nilotinib was continued without dose adjustment, emollients and low dose topical corticosteroid (1\%) hydrocortisones were prescribed, and seven days later the rash disappeared.

\section{Discussion}

Nilotinib is a second-generation Bcr-Abl tyrosine kinase inhibitors approved for use as first-line therapy for CML or as second line for imatinib resistant CML.

Nilotinib is generally well tolerated, however, as with all TKIs; it has off-target side effects. Skin reactions are one of the common non-hematological side effects reported with Nilotinib with incidence of rash varying from $22 \%$ to $62 \%$ in clinical trials [1-3]. Other dermatological side effects include pruritus, dry skin and rarely alopecia [4-7]

In the ENESTnd trial, skin rash occurred in 31\% of patients who received Nilotinib at a dose of $300 \mathrm{mg}$ twice a day, and $36 \%$ in those taking Nilotinib at $400 \mathrm{mg}$ twice a day, yet only $11 \%$ in patients taking Imatinib (Grade $3-4$ in $<1 \%$ vs. $3 \%$ vs. $1 \%$, respectively) [7]. Similarly in the MDACC trial, rash was reported in $49 \%$ of patients [3].

Skin rashes associated with Nilotinib are usually mild or moderate in severity and can present with various manifestations including erythema, morbiliform eruptions or keratosis pilarislike lesions. The lesions are usually located on the trunk and arms, and less frequently on the extremities and the face [8].

The mechanism of these skin reactions is not clear, however, it might be attributed to the tyrosine kinase inhibition action of Nilotinib and the activity of tyrosine kinases in the skin. Nilotinib inhibits the following kinases, in order of potency: Discoidin domain receptor 1 DDR-1>DDR-2>Bcr-Abl>PDGFR-A/B > c-KIT $>$ CSF-1R [9]. It also decreases extracellular matrix protein synthesis in fibroblasts derived from human skin biopsies [10].

Management usually includes topical emollients and steroids, and in some cases systemic therapies with antihistamines or short courses of systemic steroids. In mild and moderate cases, interruption or discontinuation of TKI treatment are not indicated [11].

In our cases, the rashes improved after approximately 7 days with the use of emollients and low dose topical corticosteroid without interruption or reduction of Nilotinib dose.

\section{Conclusion}

Nilotinib is an effective tyrosine kinase inhibitor for the treatment of CML, early recognition and understanding of nonhematological adverse effects including skin rashes are required for the optimal management of patients with CML as interrupting the TKI may not be in the best interest of the patient as achieving molecular remission is the goal of therapy in the management of patients with CML.

\section{References}

1. Rosti G, Palandri F, Castagnetti F, Breccia M, Levato L, Gugliotta G, Capucci A, et al. Nilotinib for the frontline treatment of $\mathrm{Ph}(+)$ chronic myeloid leukemia. Blood. 2009;114(24):4933-4938. doi: 10.1182/ blood-2009-07-232595 
2. Baccarani M, Cortes J, Pane F, Niederwieser D, Saglio G, Apperley J, Cervantes F, et al. Chronic myeloid leukemia: An update of concepts and management recommendations of European LeukemiaNet. J ClinOncol. 2009;27(35):6041-6051. doi: 10.1200/JC0.2009.25.0779

3. Cortes JE, Jones D, O’Brien S, Jabbour E, Konopleva M, Ferrajoli A, Kadia T, et al. Nilotinib as front-line treatment for patients with chronic myeloid leukemia in early chronic phase. J ClinOncol. 2010;28(3):392-397. doi: 10.1200/JC0.2009.25.4896

4. Kantarjian HM, Giles F, Gattermann N, Bhalla K, Alimena G, Palandri F, Ossenkoppele GJ, et al. Nilotinib (formerly AMN107), a highly selective BCR-ABL tyrosine kinase inhibitor, is effective in patients with Philadelphia chromosome-positive chronic myelogenous leukemia in chronic phase following imatinib resistance and intolerance. Blood. 2007;110(10): 3540-3546.

5. Nicolini FE, Masszi T, Shen Z, Gallagher NJ, Jootar S, Powell BL et al. Expanding Nilotinib Access in Clinical Trials (ENACT), an openlabel multicenter study of oral nilotinib in adult patients with imatinib-resistant or -intolerant chronic myeloid leukemia in accelerated phase or blast crisis. Leuk Lymphoma. 2012;53:907914.

6. Kantarjian H, Giles F, Wunderle L, Bhalla K, O'Brien S, Wassmann B, Tanaka C, et al. Nilotinib in imatinib-resistant CML and Philadelphia chromosome-positive ALL. N Engl J Med. 2006;354(24):2542-2551.

7. Saglio G, Kim DW, Issaragrisil S, le CP, Etienne G, Lobo C, Pasquini $\mathrm{R}$,Clark RE, et al. Nilotinib versus imatinib for newlydiagnosed chronic myeloid leukemia. N Engl J Med. 2010;362(24):2251-2259. doi: 10.1056/NEJMoa0912614
8. Drucker AM, Wu S, Busam KJ, Berman E, Amitay-Laish I, Lacouture ME. Rash with the multitargeted kinase inhibitors nilotinib and dasatinib: Meta-analysis and clinical characterization. Eur J Haematol. 2013;90(2):142-150. doi: 10.1111/ejh.12052

9. Lombardo LJ, Lee FY, Chen P, Norris D, Barrish JC, Behnia K, Castaneda S, et al. Discovery of N-(2- chloro-6-methyl- phenyl)2-(6-(4-(2-hydroxyethyl)- piperazin- 1-yl)-2-methylpyrimidin-4ylamino)thiazole-5-carboxamide (BMS-354825), a dual Src/Abl kinase inhibitor with potent antitumor activity in preclinical assays. J Med Chem. 2004;47(27):6658-6661.

10. Akhmetshina A, Dees C, Pileckyte M, Maurer B, Axmann R, Jüngel A, Zwerina J, et al. Dual inhibition of c-abl and PDGF receptor signaling by dasatinib and nilotinib for the treatment of dermal fibrosis. FASEB J. 2008;22(7):2214-2222. doi: 10.1096/fj.07-105627

11.JL Steegmann, M Baccarani, M Breccia, Casado LF, GarcíaGutiérrez V5, Hochhaus A, Kim DW, et al. European LeukemiaNet recommendations for the management and avoidance of adverse events of treatment in chronic myeloid leukaemia. Leukemia. 2016;30(8):1648-1671. doi: 10.1038/leu.2016.104

12. Nilotinib induced skin rash in chronic myeloid leukemia patients: A case series.

13. NileshWasekar, Aniket B Mohite, Chandrakala S, Vinod P, Rajesh P, Shailesh B, Farah Jijina Dr. J C Patel Department of Hematology, KEM Hospital, Mumbai, Maharashtra, India. 